\title{
Insulin treatment enhances the myocardial angiogenic response in diabetes
}

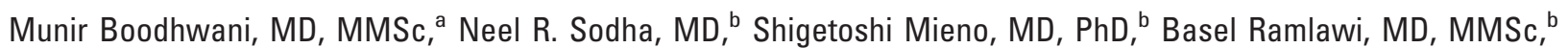
Shu-Hua Xu, PhD, ${ }^{\text {b }}$ Jun Feng, MD, PhD, ${ }^{b}$ Richard T. Clements, PhD, ${ }^{b}$ Marc Ruel, MD, MPH, ${ }^{a}$ and Frank W. Sellke, MD

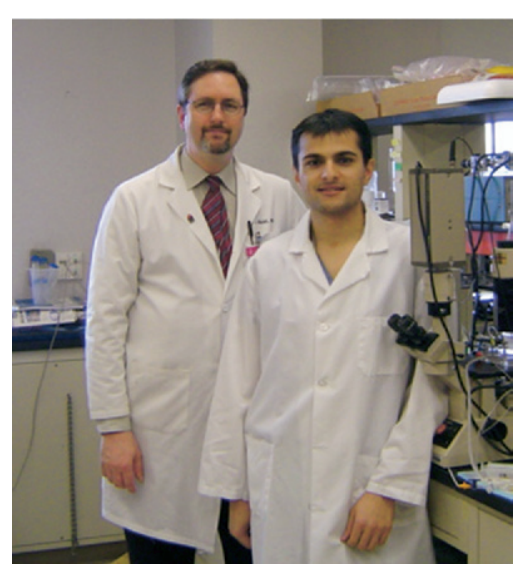

Drs Sellke and Boodhwani (left to right)
Objective: Growth factor and cell-based angiogenesis are attractive therapeutic options for diabetic patients with end-stage coronary disease. Reduced collateral vessel formation observed in diabetes is associated with increased expression of anti-angiogenic proteins, angiostatin and endostatin. The aim of this study was to determine the effects of insulin treatment on the diabetic angiogenic response to chronic myocardial ischemia.

Methods: Yucatan miniswine were treated with alloxan (pancreatic $\beta$-cell specific toxin, $150 \mathrm{mg} / \mathrm{kg}$ ) and divided into two groups. In the diabetic group (DM, $\mathrm{n}=8$ ), blood glucose levels were kept greater than $250 \mathrm{mg} / \mathrm{dL}$, and in the insulin-treated group (IDM, $\mathrm{n}=6$ ), intramuscular insulin was administered daily to keep blood glucose less than $150 \mathrm{mg} / \mathrm{dL}$. A third group of age-matched swine served as nondiabetic controls $(\mathrm{ND} ; \mathrm{n}=8)$. Eight weeks later, all animals underwent circumflex artery ameroid constrictor placement to induce chronic ischemia. Myocardial perfusion was assessed at 3 and 7 weeks after ameroid placement using microspheres. Microvascular function, capillary density, and myocardial expression of anti-angiogenic mediators were evaluated.

Results: Diabetic animals exhibited significant impairments in endothelium-dependent microvessel relaxation to adenosine diphosphate and substance $\mathrm{P}$, which were reversed in insulin-treated animals. Collateral-dependent perfusion in the ischemic circumflex territory, which was profoundly reduced in diabetic animals $(-0.18 \pm$ $\left.0.02 \mathrm{vs}+0.23 \pm 0.07 \mathrm{~mL} \cdot \mathrm{min}^{-1} \cdot \mathrm{g}^{-1} ; P<.001\right)$, improved significantly with insulin treatment $\left(0.12 \pm 0.05 \mathrm{~mL} \cdot \min ^{-1} \cdot \mathrm{g}^{-1} ; P<.01\right)$. Myocardial expression of anti-angiogenic proteins, angiostatin and endostatin, showing a 4.3- and 3.6-fold increase in diabetic animals respectively (both $P<.01$ vs ND), was markedly reduced in insulin-treated animals $(2.3-$ and 1.8 -fold vs ND; both $P<.01)$.

Conclusions: Insulin treatment successfully reversed diabetic coronary endothelial dysfunction and significantly improved the endogenous angiogenic response. These pro-angiogenic effects may be mediated through downregulation of anti-angiogenic mediators. Insulin therapy appears to be a promising modality to enhance the angiogenic response in diabetic patients.

$\mathrm{F}$ or more than a decade, cardiovascular researchers and clinicians have explored therapeutic angiogenesis, using growth factors or cell-based therapies, as a treatment option for patients with coronary artery disease. Despite early success in animal models, clinical trials of angiogenic therapies have yielded disappointing results. ${ }^{1,2}$ One of the proposed explanations for the discordance between animal studies and clinical trials is the presence of various anti-angiogenic influences that are found in patients with coronary artery disease but are absent in young healthy animals in which the majority of preclinical experiments are conducted. These include, among others, diabetes, hypercholesterolemia, advanced age, 


\author{
Abbreviations and Acronyms \\ ADP $=$ adenosine 5'-diphosphate \\ Ang-1 = angiopoiten-1 \\ $\mathrm{DM}=$ diabetes group \\ $\mathrm{dP} / \mathrm{dt}=$ first derivative of left ventricular pressure \\ IDM = insulin-treated diabetes group \\ $\mathrm{ND}=$ no diabetes control group \\ VEGF $=$ vascular endothelial growth factor
}

and endothelial dysfunction. We have previously demonstrated that diet-induced hypercholesterolemia is associated with endothelial dysfunction as well as an impairment in the endogenous $^{3}$ and growth factor-induced angiogenic response. ${ }^{4,5}$ Advanced age has been associated with impaired growth factor signaling in patients with coronary artery disease. $^{6}$

Diabetes is a commonly found comorbid condition in patients with coronary artery disease and is increasing in prevalence worldwide. ${ }^{7}$ Its presence is associated with accelerated atherosclerosis as well as an impaired angiogenic response to chronic ischemia, which has been demonstrated in patients, both angiographically ${ }^{8}$ and in autopsy studies. ${ }^{9}$ Although therapeutic angiogenesis appears to be an attractive treatment option in patients with diabetes, the evaluation of therapeutic modalities in the presence of diabetes is limited, in large part, by the lack of a large animal model of diabetes and chronic ischemia that can be used to evaluate clinically relevant end points. $\mathrm{We}^{10}$ have recently reported the creation and thorough validation of such a model of alloxan-induced diabetes in Yucatan miniswine. In this model, diabetes is induced with alloxan, a pancreatic $\beta$-cell specific toxin. We demonstrated that a 15-week exposure to chronic hypoinsulinemic hyperglycemia (type I diabetes) was associated with many of the functional, microvascular, and molecular abnormalities associated with human diabetes, including endothelial dysfunction, impaired collateraldependent myocardial perfusion, and alterations in the angiogenic signaling in the myocardium. In this study, we sought to determine the effects of glycemic control using parenteral insulin treatment on microvascular function and the endogenous angiogenic response to chronic myocardial ischemia.

\section{Materials and Methods}

\section{General Experimental Sequence and Diabetes Induction}

Twenty-two Yucatan miniswine of male sex (Sinclair Research Inc, Colombia, Mo) were used for the studies. Diabetes was induced when animals were 8 months old by a single intravenous injection of alloxan $(150 \mathrm{mg} / \mathrm{kg})$. Only animals that achieved and maintained blood glucose levels greater than $200 \mathrm{mg} / \mathrm{dL}$ were included in the diabetes group. Animals were fed ad libitum and no dietary modification was used. These animals were divided into two groups. In the diabetic group (DM, $n=8$ ), fasting blood sugar was maintained between 250 and $400 \mathrm{mg} / \mathrm{dL}$. In the insulin-treated group (IDM, $\mathrm{n}=6$ ), glycemic control was achieved (fasting blood sugar $<150 \mathrm{mg} / \mathrm{dL})$ through daily intramuscular insulin $(70 \%$ amorphous, $30 \%$ crystalline) administration. Fasting blood glucose was monitored every 2 days early after diabetes induction and 1 to 2 times per week thereafter by obtaining a small blood sample (50 $\mu \mathrm{L}$ ) from a capillary bed in the ear. Diabetes was maintained for 8 weeks before surgical instrumentation. Age-matched miniswine $(\mathrm{ND}, \mathrm{n}=8)$ served as controls.

All animals underwent an identical experimental protocol involving three separate procedures on each animal. Anesthesia was performed as reported previously, ${ }^{3}$ and animals received humane care in compliance with the Harvard Medical Area Institutional Animal Care and Use Committee and the National Research Council's "Guide for the Care and Use of Laboratory Animals," prepared by the Institute of Laboratory Animals and published by the National Institutes of Health (NIH publication No.5377-3, 1996). In brief, for all surgical procedures, anesthesia was induced with ketamine $(10 \mathrm{mg} / \mathrm{kg}$ intramuscularly), thiopental $(5-10 \mathrm{mg} / \mathrm{kg}$ intravenously), and thiopental $2.5 \%$ and maintained with a gas mixture of oxygen at 1.5 to $2 \mathrm{~L} / \mathrm{min}$ and isoflurane at $0.75 \%$ to $3.0 \%$. The animals were intubated and mechanically ventilated at 12 to 20 breaths/min.

The first procedure, performed via a small left anterolateral thoracotomy 8 weeks after diabetes induction, consisted of the placement of a $1.75-\mathrm{mm}$ ameroid constrictor around the proximal circumflex artery and the injection of $1.5 \times 10^{7}$ gold-labeled microspheres into the left atrium during temporary occlusion of the circumflex coronary artery, to subsequently allow for identification, by shadow labeling, of the myocardial territory at risk.

The second procedure, also performed via left anterolateral thoracotomy, 3 weeks after ameroid placement, consisted of $1.5 \times$ $10^{7}$ lutetium microspheres injected in the left atrium during rest conditions and $1.5 \times 10^{7}$ europium microspheres injected during rapid atrial pacing ( 150 beats $/ \mathrm{min}$ ) to allow for determination of baseline perfusion after ameroid closure. To document ameroid closure, we performed left coronary angiography through an $8 \mathrm{~F}$ sheath (Cordis Corporation, Miami, Fla) surgically inserted in the femoral artery, using a catheter with the appropriate distal angulation and high atomic weight contrast (Mallinckrodt Inc, St Louis, Mo).

The third procedure was carried out 4 weeks after the second procedure and 7 weeks after ameroid placement. Sternotomy was performed, $1.5 \times 10^{7}$ samarium microspheres were injected into the left atrium during rest conditions, and $1.5 \times 10^{7}$ lanthanum microspheres were injected during pacing (150 beats/min). The animals were then humanely killed with $10 \mathrm{~mL} / \mathrm{kg}$ of a saturated $\mathrm{KCl}$ solution administered intravenously. Cardiac samples were harvested and snap frozen for molecular studies, sectioned, weighed, and dried for myocardial microsphere analyses, and put in $4^{\circ} \mathrm{C}$ Krebs solution for in vitro assessment of coronary microvascular reactivity. Ameroid constrictors were resected along with a segment of circumflex artery and examined under low-power magnification to confirm occlusion. 


\section{In Vitro Assessment of Coronary Microvessel Reactivity}

After cardiac harvest, epicardial coronary arterioles (80-150 $\mu \mathrm{m}$ in diameter and 1-2 $\mathrm{mm}$ in length) originating from branches of the left anterior descending and circumflex arteries were dissected from the surrounding tissue with a $\times 40$ dissecting microscope and examined in isolated organ chambers, as described previously. ${ }^{11}$ The responses to sodium nitroprusside ( $1 \mathrm{nmol}-100 \mu \mathrm{mol})$, an endothelium-independent cyclic guanosine monophosphatemediated vasodilator, as well as adenosine $5^{\prime}$-diphosphate (ADP) ( $1 \mathrm{nmol} / \mathrm{L}-10 \mu \mathrm{mol} / \mathrm{L})$, substance $\mathrm{P}(1 \mathrm{fmol} / \mathrm{L}-1 \mathrm{nmol} / \mathrm{L})$, and vascular endothelial growth factor (VEGF, $1 \mathrm{fmol} / \mathrm{L}-1 \mathrm{nmol} / \mathrm{L}$ ), three endothelium-dependent receptor-mediated vasodilators that act via bioavailable nitric oxide, were studied after precontraction by $20 \%$ to $50 \%$ of the baseline diameter with the thromboxane $A_{2}$ analog U46619 (0.1-1 $\mu \mathrm{mol} / \mathrm{L})$. Relaxation responses were defined as the percent relaxation of the precontracted diameter, and 6 to 8 vessels were examined from 6 to 8 animals in each group from the left anterior descending and the circumflex territories.

\section{Assessment of Myocardial Perfusion}

Myocardial perfusion was assessed during each procedure with isotope-labeled microspheres (BioPAL, Worcester, Mass) using methods previously reported. ${ }^{3}$ Isotope-labeled microspheres, 15 $\mu \mathrm{m}$ in diameter, of different isotopic mass were used at each experimental stage. Gold-labeled microspheres were injected during temporary circumflex occlusion at the time of ameroid placement to identify myocardial samples that originated from the circumflex coronary distribution (those with the lowest count of gold-labeled microspheres). Lutetium- and europium-labeled microspheres were used during the second procedure to determine baseline blood flow at rest and with atrial pacing at 150 beats/min. Samarium- and lanthanum-labeled microspheres were injected at rest and during pacing during the third procedure. Reference blood samples were obtained from the femoral artery during the second and third procedures. After the animals were humanely killed, 10 circumferential, transmural left ventricular sections were collected for assay of isotope-labeled microspheres in each animal, weighed, and dried at $60^{\circ} \mathrm{C}$ for 24 hours. Each sample was exposed to neutron beams and microsphere densities were measured in a gamma counter. Adjusted myocardial blood flow (at rest and with pacing), reflecting changes in lateral myocardial perfusion, was determined from the two myocardial samples that showed the lowest count of gold microspheres by the following equations:

Crude blood flow (tissue sample) $=$

(Withdrawal rate $[\mathrm{mL} / \mathrm{min}] /$ weight [tissue sample])

$\times$ (Isotope counts [tissue sample]/

Isotope counts [reference blood sample])

Adjusted blood flow $=$ Crude blood flow (third operation)

- Crude blood flow at baseline (second operation)

\section{Assessment of Myocardial Function}

Before tissue harvest, a catheter was surgically inserted through the femoral artery, passed into the left ventricle, and left ventricular pressure was recorded over 10-second intervals. Global systolic and diastolic function was determined with Cardiosoft soft- ware (Sonosoft Inc, London, Ontario, Canada) by taking the first derivative of left ventricular pressure $(\mathrm{dP} / \mathrm{dt})$. Next, maximum $+\mathrm{dP} / \mathrm{dt}$ (systolic function) and minimum $-\mathrm{dP} / \mathrm{dt}$ (diastolic function) measurements were obtained and averaged over 15 to 20 cardiac cycles in each animal.

\section{Immunohistochemistry}

Myocardial sections from the circumflex territory of control and diabetic animals were stained with anti-platelet-endothelial cell adhesion molecule-1 (CD-31) antibody diluted to 1:600 (BD Biosciences, San Diego, Calif) as previously described. ${ }^{3}$ The sections were counterstained with methyl green and examined for capillary endothelial cell density in a triplicate, blinded fashion from $600 \times$ $440 \mu \mathrm{m}\left(0.264 \mathrm{~mm}^{2}\right)$ cross-sectional fields randomly selected from the center of circumflex territories.

\section{Western Blotting}

Whole-cell lysates were isolated from the homogenized myocardial samples with a radioimmunoprecipitation assay buffer (Boston Bioproducts, Worcester, Mass) and centrifuged at $12,000 \mathrm{~g}$ for 10 minutes at $4^{\circ} \mathrm{C}$ to separate soluble from insoluble fractions. Protein concentration was measured spectrophotometrically at a 595-nm wavelength with a DC protein assay kit (Bio-Rad Laboratories, Hercules, Calif). Forty to eighty micrograms of total protein were fractionated by $4 \%$ to $20 \%$ gradient, sodium dodecylsulfate polyacrylamide gel electrophoresis (Invitrogen, San Diego, Calif) and transferred to polyvinyl difluoride membranes (Millipore, Bedford, Mass). Each membrane was incubated with specific antibodies as follows: anti-VEGF antibody (dilution 1:250) (Calbiochem, San Diego, Calif), anti-endothelial nitric oxide synthase antibody (1:2500) (BD Biosciences, San Jose, Calif), anti-Tie-2 antibody (1:200) (Santa Cruz, Santa Cruz, Calif), anti-endostatin antibody (1:1000) (Upstate, Chicago, Ill), and antiangiostatin antibody. Then the membranes were incubated for 1 hour in diluted appropriate secondary antibody (Jackson Immunolab, West Grove, Pa). Immune complexes were visualized with the enhanced chemiluminescence detection system (Amersham, Piscataway, NJ). Bands were quantified by densitometry of radioautograph films.

\section{Data Analysis}

Data are reported as means \pm SEM. Microvessel responses are expressed as percent relaxation of the preconstricted diameter and were analyzed by 2-way repeated measures analysis of variance, examining the relationship between vessel relaxation, log concentration of the vasoactive agent of interest, and the experimental group (SAS version 9.1; SAS Institute, Inc, Cary, NC). Immunoblots are expressed as a ratio of protein to loading band density and were analyzed after digitization and quantification of x-ray films with ImageJ 1.33 (National Institutes of Health, Bethesda, Md). Blots and isotope-labeled microsphere data were analyzed by analyses of variance. Bonferroni corrections were applied to multiple tests.

\section{Results}

Experimental Model

Diabetes induction was attempted in 17 animals, of which $14(82 \%)$ demonstrated consistent blood glucose levels 


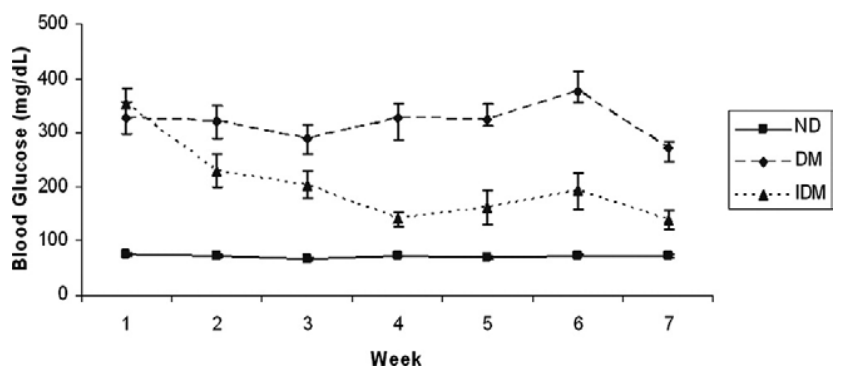

Figure 1. Blood glucose levels in no diabetes (ND), diabetes (DM), and insulin-treated diabetes (IDM) groups over the 7-week experimental protocol.

greater than $200 \mathrm{mg} / \mathrm{dL}$. One animal died of renal failure and 2 failed to become hyperglycemic. Four animals in the DM group occasionally required low doses of insulin (10-20 u/day) to keep blood sugar levels less than $400 \mathrm{mg} / \mathrm{dL}$. In the IDM group, all animals required large doses of daily insulin (average dose of $78 \mathrm{U} /$ day) to achieve target fasting blood glucose levels. Three of the 6 IDM animals required twice a day dosing to achieve adequate glycemic control. Hypoglycemic episodes requiring treatment occurred in 2 animals on 4 occasions. Fasting blood glucose levels in the different groups throughout the experimental protocol are depicted in Figure 1. All animals survived the entire experimental protocol.

\section{Coronary Microvessel Reactivity}

Results of microvessel relaxation studies are summarized in Figure 2. U46619 was used at a dose of $1 \times 10^{-6} \mathrm{~mol} / \mathrm{L}$ to preconstrict coronary microvessels by an average of $40 \% \pm$ $4 \%$ of the baseline diameter. Compared with the control group (ND), diabetic animals (DM and IDM) demonstrated an impairment in microvessel relaxation to endotheliumdependent vasorelaxants, ADP and substance $\mathrm{P}$ (both $P<$
.001 ), in the ischemic territory, suggesting reduced nitric oxide bioavailability. IDM animals showed almost complete recovery of this endothelial dysfunction. Furthermore, microvessel relaxation in response to VEGF was markedly impaired in diabetic animals $(P<.001)$. IDM animals demonstrated significant improvement in the microvascular response to VEGF compared with the DM group $(P<.01)$ but still remained impaired compared with controls $(P<$ $.05)$. Endothelium-independent relaxation, in response to sodium nitroprusside, was not significantly different between groups $(P=.09)$.

\section{Myocardial Perfusion}

Crude circumflex territory myocardial blood flow was similar between control, DM, and IDM groups at 3 weeks after ameroid placement, both at rest $(0.43 \pm 0.07,0.33 \pm 0.02$, and $0.34 \pm 0.04 \mathrm{~mL} \cdot \min ^{-1} \cdot \mathrm{g}^{-1}$, respectively; $\left.P=.10\right)$ and with pacing $(0.30 \pm 0.04$ vs $0.39 \pm 0.03$ vs $0.28 \pm 0.08$ $\left.\mathrm{mL} \cdot \min ^{-1} \cdot \mathrm{g}^{-1} ; P=.25\right)$. At 7 weeks, the control animals had an increase in circumflex territory perfusion at rest $\left(+0.23 \pm 0.07 \mathrm{~mL} \cdot \mathrm{min}^{-1} \cdot \mathrm{g}^{-1}\right)$, whereas the DM animals demonstrated a reduction in perfusion $(-0.18 \pm 0.02 \mathrm{~mL}$. $\left.\min ^{-1} \cdot \mathrm{g}^{-1} ; P<.001\right)$. Insulin-treated diabetic animals (IDM) demonstrated significantly improved perfusion compared with DM animals $\left(0.12 \pm 0.05 \mathrm{~mL} \cdot \mathrm{min}^{-1} \cdot \mathrm{g}^{-1} ; P\right.$ $<.001)$ but remained significantly lower than control animals $(P<.05)$. These differences were also evident during rapid atrial pacing (Figure 3).

\section{Myocardial Function}

Global left ventricular function was measured at the time of the final procedure, 7 weeks after ameroid placement, and systolic and diastolic function were quantified using maximum positive $\mathrm{dP} / \mathrm{dt}$ and minimum negative $\mathrm{dP} / \mathrm{dt}$, respectively. Diabetic animals demonstrated reduced left ventricular contractility $(P<.001)$, which was significantly
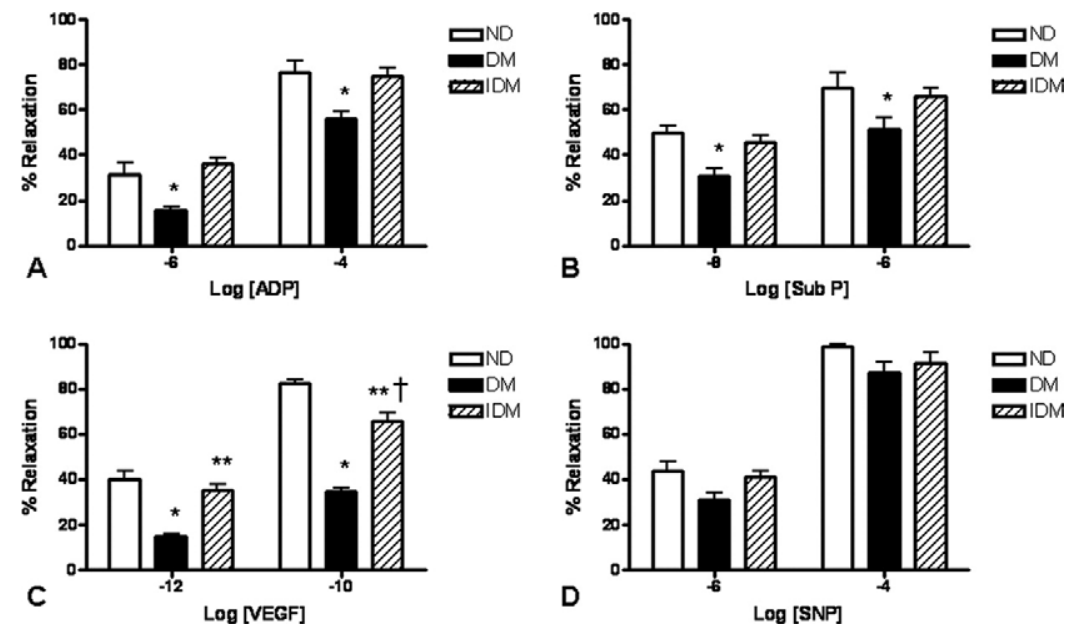

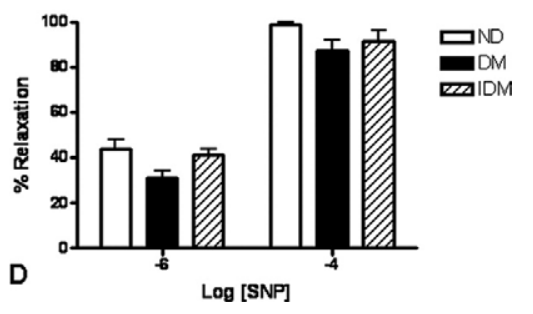

Figure 2. Coronary microvascular reactivity. Percent relaxation to increasing concentrations of vasodilating agents after preconstriction with U46619. Responses to endothelium-dependent vasodilators ADP (A), Substance $P(B)$, and VEGF (C), as well as endothelium-independent vasodilator SNP (D) in the ischemic territory of ND ( $n=$ 8), DM ( $n=8)$, and IDM ( $n=6)$ animals. ADP, Adenosine 5' diphosphate; SNP, sodium nitroprusside; VEGF, vascular endothelial growth factor. ${ }^{*} P<.001$ vs ND. ${ }^{* *} P<.01$ vs $D M .+P<.05$ vs controls. ND, DM, and IDM as in Figure 1. 

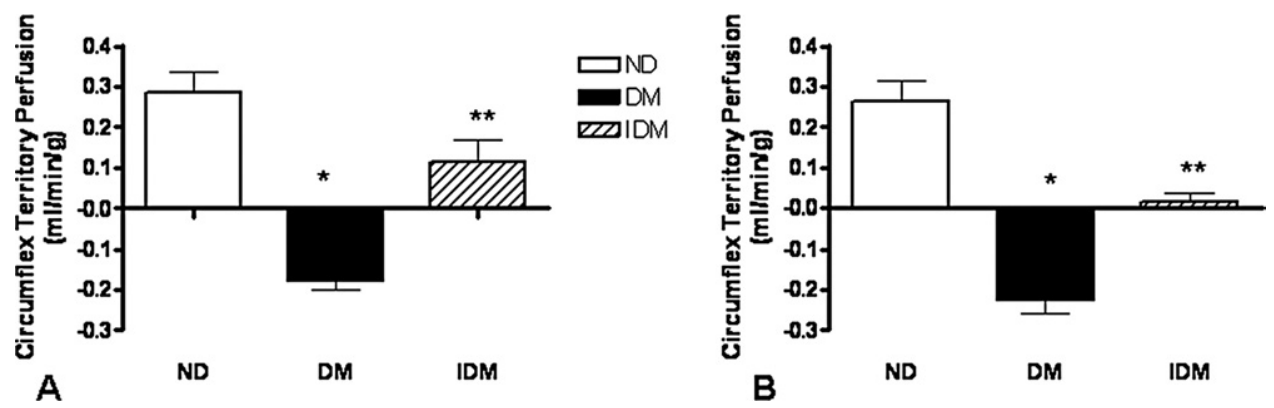

Figure 3. Myocardial perfusion. Isotope-labeled microspheres were used to determine baseline-adjusted circumflex territory myocardial blood perfusion in control $(n=8)$ and diabetic swine (DM, $n=8$; IDM, $n=6)$ after circumflex ameroid occlusion. Adjusted circumflex territory perfusion, which was profoundly reduced in DM animals at rest $(A)$ and with pacing $(B)$, improved with insulin treatment, but still remained lower than controls. ${ }^{*} P<.001$ vs ND. ${ }^{* *} P<.001$ vs DM and $P<.05$ vs ND. ND, DM, and IDM as in Figure 1 .

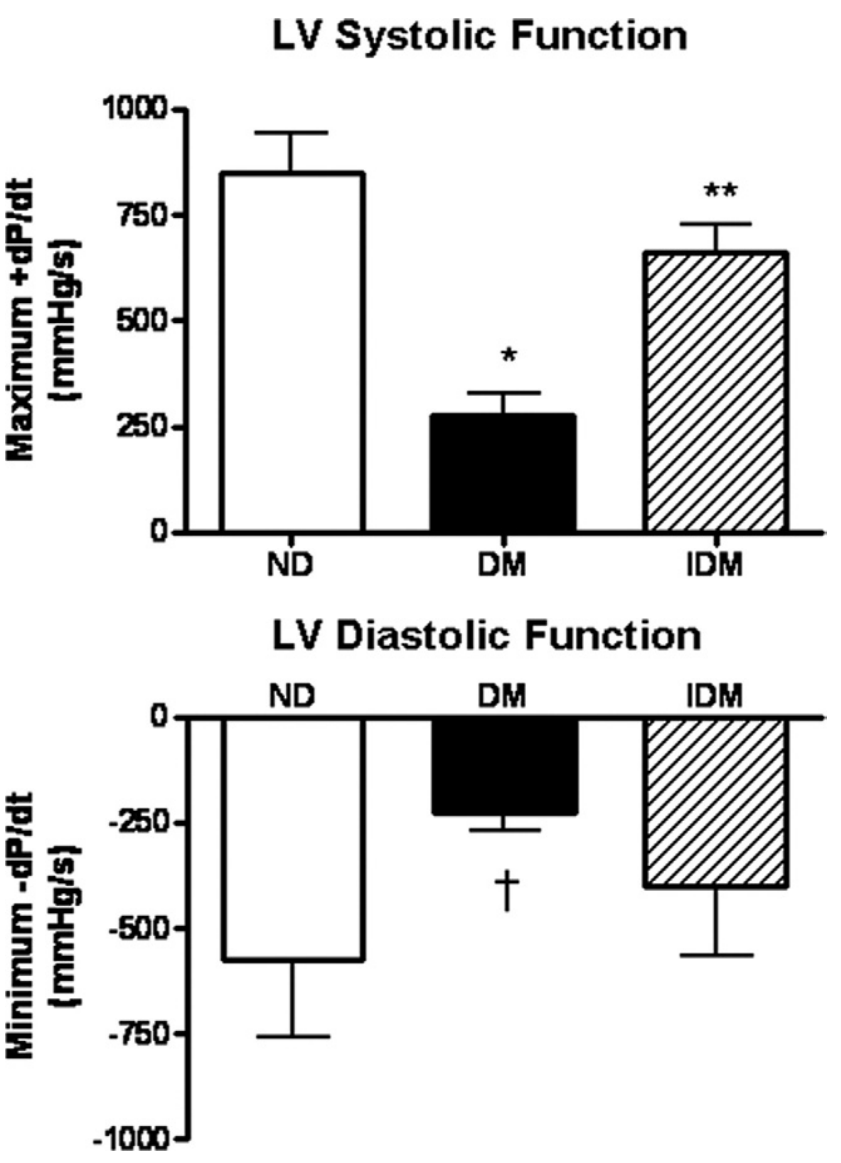

Figure 4. Left ventricular ( $L V$ function. Diabetic animals demonstrated reduced LV contractility and impaired LV relaxation. Insulin treatment resulted in significant improvement in LV systolic function (n = 4-6/group; ${ }^{*} P<.001$ vs ND; ${ }^{*} P<.01$ vs $D M ; ~+P<$ .05 vs ND). ND, DM, and IDM as in Figure 1. improved with insulin treatment $(P<.01)$. Similar abnormalities were observed with respect to diastolic function in diabetic animals $(P<.05)$. However, insulin treatment did not result in a statistically significant difference (Figure 4).

\section{Capillary Endothelial Cell Density}

Figure 5 shows the density of CD31+ capillary endothelial cells (cells/high power field) in the ischemic territory of pigs from all groups 7 weeks after ameroid placement. Diabetic animals had significantly reduced endothelial cell density compared with controls $(160 \pm 10$ vs. $263 \pm 18 ; P<.001)$.

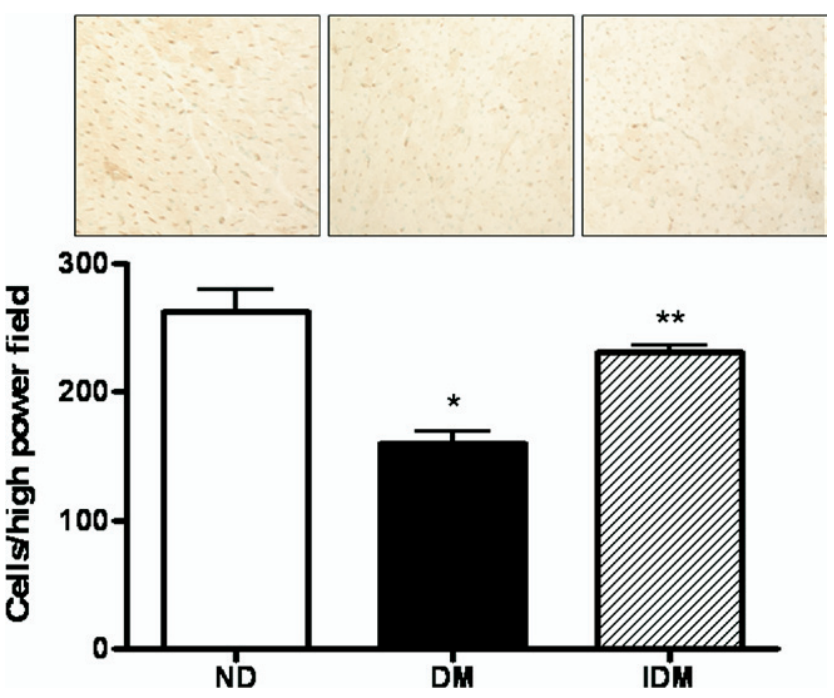

Figure 5. Endothelial cell density. CD31+ endothelial cell density per high power field $\left(0.264 \mathrm{~mm}^{2}\right)$ was determined in the ischemic territory of control ( $n=8$ ) and diabetic (DM, $n=8$; IDM, $n=6$ ) animals. Diabetic animals had reduced endothelial cell density compared with controls, which was significantly improved in insulin-treated diabetic animals. ${ }^{*} \boldsymbol{P}<.001$ vs ND. ${ }^{* *} \boldsymbol{P}<.001$ vs DM. ND, DM, and IDM as in Figure 1. 


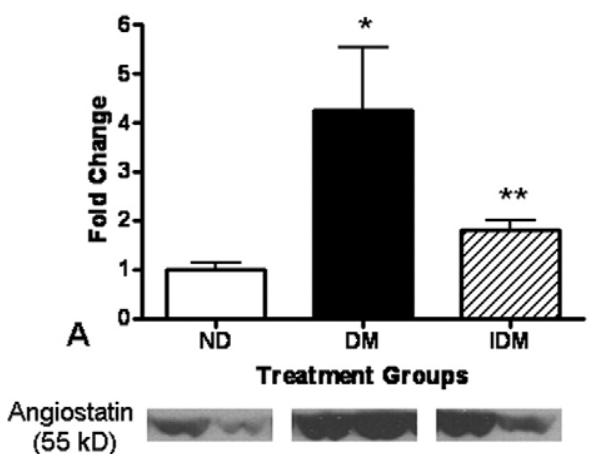

$(55 \mathrm{kD})$

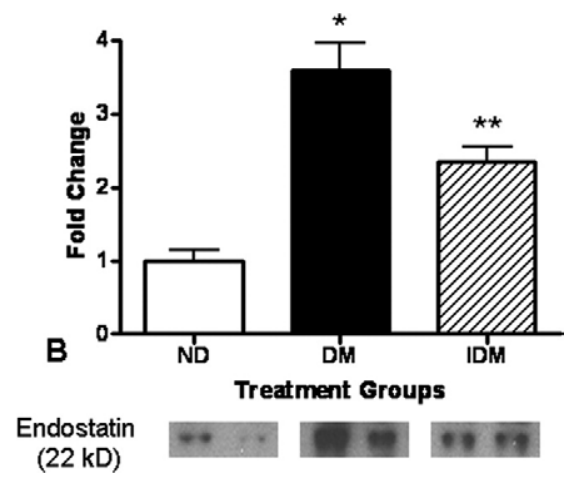

Figure 6. Myocardial expression of antiangiogenic mediators. Expression of angiostatin (A) and endostatin (B) was significantly increased in the myocardium of diabetic animals and was significantly reduced in insulin-treated diabetic animals. (n $=4-6 /$ group). ${ }^{*} \boldsymbol{P}<$ .01. ${ }^{* *} P<.05$. ND, DM, and IDM as in Figure 1.
Animals in the IDM group demonstrated significantly higher endothelial cell density than did DM animals (230 \pm 7; $P<.001$ vs DM).

\section{Western Blotting}

$\mathrm{We}^{10}$ have previously reported the effects of diabetes on the expression and activity of angiogenic mediators in the myocardium. We examined the effects of insulin treatment on pro-angiogenic and anti-angiogenic mediator expression in the diabetic myocardium.

Anti-angiogenic mediators: Angiostatin and endostatin. Figure 6 displays the results of Western blots of antiangiogenic mediators, angiostatin and endostatin. The expression of both angiostatin $(4.1 \pm 1.3$-fold; $P<.01)$ and endostatin $(3.6 \pm 0.4$-fold; $P<.01)$ was profoundly increased in the myocardium of diabetic animals. IDM animals demonstrated a significant reduction in myocardial angiostatin $(1.8 \pm 0.2$-fold vs ND; $P<.05$ for comparison of IDM vs DM) and endostatin $(2.3 \pm 0.2$-fold vs ND; $P<$ .01 for comparison of IDM vs DM) expression. Similar patterns of expression of the anti-angiogenic proteins were observed in both the ischemic circumflex and the nonischemic territories of the left anterior descending coronary artery.

Pro-angiogenic mediators: VEGF and angiopoietin pathways. VEGF and angiopoiten-1 (Ang-1) are important pro-angiogenic growth factors that mediate their effects through binding to their respective receptors, VEGFR2 and Tie-2. The DM group had significantly lower myocardial expression of growth factors, VEGF and Ang-1 (0.60 \pm 0.11 -fold and $0.62 \pm 0.06$-fold vs ND, respectively; both $P$ $<$.05). Insulin treatment in the IDM animals led to significant increases in VEGF and Ang-1 levels compared with the DM group $(1.17 \pm 0.08$-fold and $1.03 \pm 0.08$-fold vs ND, respectively; both $P<.05$ vs DM). VEGFR2 levels were similar in all groups. Tie-2 expression was similar between ND and DM animals but significantly increased in IDM animals $(2.3 \pm 0.2$-fold vs ND; $P<.001)$. Expression of downstream mediator endothelial nitric oxide synthase was similar between groups (Figure 7).

\section{Discussion}

In a clinically relevant model of diabetes and chronic myocardial ischemia, we sought to evaluate the effects of glycemic control using insulin on the endogenous angiogenic response. We found that insulin treatment was successful in achieving the target fasting blood glucose level of less than $150 \mathrm{mg} / \mathrm{dL}$ and associated with reversal of the endothelial dysfunction observed in diabetic animals. VEGF signaling

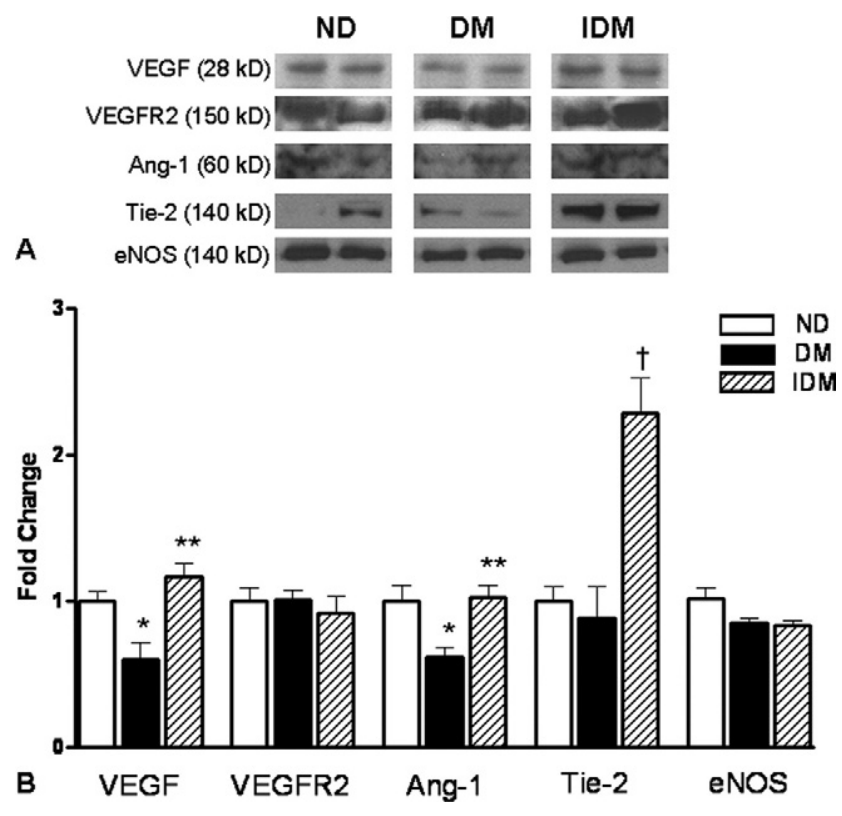

Figure 7. Myocardial expression of pro-angiogenic mediators. A, Expression of growth factors, VEGF and Ang-1, their respective receptors, VEGFR2 and Tie-2, as well as downstream mediator eNOS were assessed. B, Quantitative analysis showed that diabetic animals had reduced myocardial expression of VEGF and Ang-1, but VEGFR2 and Tie-2 expression was unchanged. Insulin treatment caused an increase in VEGF and Ang-1 expression as well as a significant increase in Tie-2 expression. Ang-1, Angiopoiten-1; VEGF, vascular endothelial growth factor; eNOS, endothelial nitric oxide synthase ( $n=4-6 /$ group). ${ }^{*} P<.05$ v. ND. ${ }^{* *} P$ $<.05$ vs $D M ;+P<.001$ vs ND. ND, DM, and IDM as in Figure 1. 
in coronary microvessels, which was significantly impaired in diabetic animals, recovered only partially with insulin treatment. Perfusion of the collateral dependent territory was profoundly diminished in diabetic animals. Insulin treatment led to a significant improvement but did not normalize the perfusion of the ischemic myocardium. This was corroborated by the morphologic evidence of improved endothelial cell density in insulin-treated animals and was also associated with improvement in global left ventricular systolic function. Exploration of pro-angiogenic and antiangiogenic pathways revealed that the profound increases in the expression of anti-angiogenic proteins, angiostatin and endostatin, observed with diabetes, were significantly reduced in insulin-treated animals. Furthermore, the expression of pro-angiogenic growth factors, VEGF and Ang-1, as well as the receptor Tie-2, was enhanced with insulin treatment. In summary, this study provides functional, morphologic, microvascular, and molecular evidence for the proangiogenic effects of glycemic control achieved with insulin treatment in the setting of diabetes.

Glycemic control remains the mainstay of treatment in diabetes and has been shown to improve both macrovascular and microvascular complications of diabetes. ${ }^{12,13}$ Insulin treatment, with or without oral hypoglycemic agents, is the most common method used clinically to achieve glycemic control. Insulin has multifaceted effects on the myocardium, which mainly involve the regulation of fuel consumption, glucose transport, glycogen synthesis, and glycolysis. ${ }^{14}$ More relevant to vascular function, however, is the demonstrated ability of insulin to increase endothelial nitric oxide availability in the vasculature. ${ }^{15}$ Furthermore, the insulin receptor, which is present in the myocardium, is a tyrosine kinase receptor that shares many of the downstream mediators common to angiogenic growth factors and their receptors, for example PI3 kinase and mitogen-activated protein kinases. ${ }^{15}$ In addition to its direct effects on the myocardium and coronary vasculature, insulin exerts indirect effects through the reduction in systemic blood glucose levels. By reducing blood glucose levels, insulin can avoid the adverse effects of chronic hyperglycemia, which include increased oxidative stress, chronic inflammation, and the nonenzymatic glycation of proteins, particularly in the extracellular matrix. ${ }^{16,17}$ The pro-angiogenic effects of insulin demonstrated in this study may be due to a combination of the aforementioned processes.

We found that although endothelium-independent microvessel relaxation to sodium nitroprusside was preserved in all groups, diabetes was associated with impaired microvascular response to ADP and substance $\mathrm{P}$, implying endothelial dysfunction and reduced nitric oxide bioavailability. Treatment with insulin led to a complete reversal in this endothelial dysfunction. However, the impairment in VEGF-induced microvessel relaxation, observed in diabe- tes, was only partially improved in insulin-treated animals, suggesting a persistent, residual impairment in VEGF signaling in the coronary microvasculature. Examination of pro-angiogenic proteins at the molecular level revealed that expression of VEGF, Ang-1, and its receptor, Tie-2, was markedly enhanced with insulin treatment.

Angiostatin and endostatin, cleavage products of plasminogen and collagen XVIII, respectively, have been shown to have potent anti-angiogenic effects in vitro and in rodent models. ${ }^{18,19}$ Weihrauch and associates ${ }^{20}$ have associated increased angiostatin with hyperglycemia and impaired angiogenesis in dogs. The association between angiostatin and diabetes and diminished coronary collateral formation has also been shown in patients with coronary disease. $^{21,22} \mathrm{We}^{10}$ have previously shown that both angiostatin and endostatin are upregulated many fold in the setting of diabetes. In this study, we found that insulin treatment caused a significant reduction but not complete normalization in angiostatin and endostatin expression. The expression of these anti-angiogenic mediators mirrored the functional finding of significantly improved but not normalized perfusion of the ischemic circumflex territory. These observations provide further evidence for the critical role of these anti-angiogenic mediators, both as markers and as potential novel targets for the modulation of the angiogenic response in diabetes. Furthermore, the effects of insulin on matrix metalloproteinases, which are responsible for the production of these anti-angiogenic proteins, needs to be further evaluated in this setting.

\section{Model Strengths and Limitations}

Our model of alloxan-induced diabetes largely mimics type I diabetes, that is, hypoinsulinemic hyperglycemia. Although various pathophysiologic and molecular aspects of the disease are common between type I and type II diabetes ${ }^{23}$ including chronic hyperglycemia, endothelial dysfunction, increased oxidative stress and inflammation, accelerated atherosclerosis, and diminished angiogenesis, other features may be different and may limit the generalizability of this model to type II diabetes and insulin resistance. Last, whereas this model of chronic ischemia provides physiologically relevant measures of perfusion as well as morphologic assessment of angiogenesis, it is limited by the fact that molecular markers are only assessed at a single time point, 7 weeks after ameroid placement, and therefore, acute changes in pro-angiogenic and anti-angiogenic mediators may not be completely captured in this model.

\section{Conclusions}

Insulin treatment, targeted to achieve glycemic control, reverses coronary endothelial dysfunction and significantly improves but does not normalize the endogenous myocardial angiogenic response to chronic ischemia in the setting 
of diabetes. These effects may be mediated by the direct effects of insulin on the myocardium and coronary vasculature or indirectly through systemic reductions in blood glucose. Anti-angiogenic proteins, angiostatin and endostatin, are downregulated with insulin treatment and appear to play an important role in the diabetic angiogenic response. Insulin therapy appears to be a promising strategy to improve the results of growth factor or cell-based angiogenic therapies in diabetic patients.

\section{References}

1. Simons M, Bonow RO, Chronos NA, Cohen DJ, Giordano FJ, Hammond $\mathrm{HK}$, et al. Clinical trials in coronary angiogenesis: issues, problems, consensus-an expert panel summary. Circulation. 2000; 102:E73-86.

2. Boodhwani M, Sodha NR, Laham RJ, Sellke FW. The future of therapeutic myocardial angiogenesis. Shock. 2006;26:332-41.

3. Boodhwani M, Nakai Y, Mieno S, Voisine P, Bianchi C, Araujo EG, et al. Hypercholesterolemia impairs the myocardial angiogenic response in a swine model of chronic ischemia: role of endostatin and oxidative stress. Ann Thorac Surg. 2006;81:634-41.

4. Ruel M, Wu GF, Khan TA, Voisine P, Bianchi C, Li J, Laham RJ, et al. Inhibition of the cardiac angiogenic response to surgical FGF-2 therapy in a swine endothelial dysfunction model. Circulation. 2003; 108(Suppl 1):II335-40.

5. Voisine P, Bianchi C, Ruel M, Malik T, Rosinberg A, Feng J, et al. Inhibition of the cardiac angiogenic response to exogenous vascular endothelial growth factor. Surgery. 2004;136:407-15.

6. Mieno S, Boodhwani M, Clements RT, Ramlawi B, Sodha NR, Li J, et al. Aging is associated with an impaired coronary microvascular response to vascular endothelial growth factor in patients. $J$ Thorac Cardiovasc Surg. 2006;132:1348-55.

7. Zimmet P, Alberti KG, Shaw J. Global and societal implications of the diabetes epidemic. Nature. 2001;414:782-7.

8. Abaci A, Oguzhan A, Kahraman S, Eryol NK, Unal S, Arinc H, et al. Effect of diabetes mellitus on formation of coronary collateral vessels. Circulation. 1999;99:2239-42

9. Yarom R, Zirkin H, Stammler G, Rose AG. Human coronary microvessels in diabetes and ischaemia. Morphometric study of autopsy material. J Pathol. 1992;166:265-70.

10. Boodhwani M, Sodha NR, Mieno S, Xu S, Feng J, Ramlawi B, et al. Functional, cellular, and molecular characterization of the angiogenic response to chronic myocardial ischemia in diabetes. Circulation. In press.

11. Tofukuji M, Metais C, Li J, Hariawala MD, Franklin A, Vassileva C, et al. Effects of ischemic preconditioning on myocardial perfusion, function, and microvascular regulation. Circulation. 1998;98(19 Suppl): II197-204; discussion II204-5.

12. The effect of intensive treatment of diabetes on the development and progression of long-term complications in insulin-dependent diabetes mellitus. The Diabetes Control and Complications Trial Research Group. N Engl J Med. 1993;329:977-86.

13. Standards of medical care in diabetes. Diabetes Care. 2005;28:S4-36.

14. Brownsey RW, Boone AN, Allard MF. Actions of insulin on the mammalian heart: metabolism, pathology and biochemical mechanisms. Cardiovasc Res. 1997;34:3-24.

15. White MF. Insulin signaling in health and disease. Science. 2003;302: $1710-1$.

16. Zhang L, Zalewski A, Liu Y, Mazurek T, Cowan S, Martin JL, et al. Diabetes-induced oxidative stress and low-grade inflammation in porcine coronary arteries. Circulation. 2003;108:472-8.
17. Wautier JL, Schmidt AM. Protein glycation: a firm link to endothelial cell dysfunction. Circ Res. 2004;95:233-8.

18. Sharma MR, Tuszynski GP, Sharma MC. Angiostatin-induced inhibition of endothelial cell proliferation/apoptosis is associated with the down-regulation of cell cycle regulatory protein cdk5. J Cell Biochem. 2004:91:398-409.

19. O’Reilly MS, Boehm T, Shing Y, Fukai N, Vasios G, Lane WS, et al. Endostatin: an endogenous inhibitor of angiogenesis and tumor growth. Cell. 1997;88:277-85.

20. Weihrauch D, Lohr NL, Mraovic B, Ludwig LM, Chilian WM, Pagel PS, et al. Chronic hyperglycemia attenuates coronary collateral development and impairs proliferative properties of myocardial interstitial fluid by production of angiostatin. Circulation. 2004;109:2343-8.

21. Chung AW, Hsiang YN, Matzke LA, McManus BM, van Breemen C, Okon EB. Reduced expression of vascular endothelial growth factor paralleled with the increased angiostatin expression resulting from the upregulated activities of matrix metalloproteinase-2 and -9 in human type 2 diabetic arterial vasculature. Circ Res. 2006;99:140-8.

22. Matsunaga T, Chilian WM, March K. Angiostatin is negatively associated with coronary collateral growth in patients with coronary artery disease. Am J Physiol Heart Circ Physiol. 2005;288:H2042-6.

23. Guo M, Wu MH, Korompai F, Yuan SY. Upregulation of PKC genes and isozymes in cardiovascular tissues during early stages of experimental diabetes. Physiol Genomics. 2003;12:139-46.

\section{Discussion}

Dr Mark J. Krasna (Towson, Md). I was quite impressed with the technical feat of this study. Can you elaborate on the three procedures that you performed on each of these animals and was there any mortality among the three groups?

Dr Boodhwani. In the past we have done more than 400 ameroid constrictor experiments in our laboratory. We found that in a potent model of diet induced hypercholesteremia, there was about a $10 \%$ to $20 \%$ mortality rate with the animals, but over time the techniques have been improved and we have a much better sense of what the model entails now. Fortunately, in this study, we did not lose any animals.

Dr Krasna. Along the same lines, when you are doing three procedures on each animal, was there a significant infection rate from the first to the third, or was that also low?

Dr. Boodhwani. In the normal, nondiabetic animals (group ND) there was virtually no infection. In the diabetic animals we found that there was some impairment of wound healing and breakdown of the incision. However, to emphasize, all animals were treated with postoperative antibiotics for 5 days.

Dr Paul W. M. Fedak (Calgary, Alberta, Canada). This is a great study. I think this model is going to be very important for future studies in the area. My question to you is, I did not see anything about regional or global left ventricular function. Was that addressed in this study or will it be addressed in any future studies? If not, what would you hypothesize would be the effects of insulin treatment?

Dr Boodhwani. In the past we have demonstrated that circumflex ameroid constrictor placement leads to a reduction in perfusion as well as regional wall motion abnormalities. Regional wall motion abnormalities were not specifically studied in this model, although we did assess global left ventricular function. We expect, though, that improvement in perfusion would likely result in improvement in regional wall motion as well. 